\title{
Stabilization of Driven Pendulum with Periodic Linear Forces
}

\author{
Babar Ahmad \\ COMSATS Institute of Information Technology, Islamabad 44000, Pakistan \\ Correspondence should be addressed to Babar Ahmad; babar.sms@gmail.com
}

Received 27 March 2013; Revised 18 August 2013; Accepted 23 August 2013

Academic Editor: Huai-Ning Wu

Copyright (c) 2013 Babar Ahmad. This is an open access article distributed under the Creative Commons Attribution License, which permits unrestricted use, distribution, and reproduction in any medium, provided the original work is properly cited.

Using Kapitza method of averaging for arbitrary periodic forces, the pendulum driven by different forms of periodic piecewise linear forces is stabilized. These periodic piecewise linear forces are selected in the range $[-1,1]$ to establish an exact comparison with harmonic forces. In this contest, the rectangular force was found to be the best, but this force is more effective when it has a timedependent structure. This time-dependent structure is found by defining a parametric control on some other periodic piecewise linear forces.

\section{Introduction}

A pendulum with fixed suspension has only one stable point, while a pendulum whose suspension has fast oscillation can have more stable points (can oscillate). Such phenomena were first studied by Stephenson in 1908 [1-3]. In 1951, Kapitza presented this problem in a different way [4], so-called Kapitza pendulum. In 1960, Landau et al. studied the stability of such a system driven by harmonic force [5]. Then, its rapid growing applications started such as trapping of particles by laser [6-8], control of robotic devices $[9,10]$, effect on price equilibrium [11], and control by lasers in cybernetics [12].

Next in place of harmonic force Ahmad and Borisenok (2009) used periodic kicking forces, modifying Kapitza method for arbitrary periodic forces [13]. Also, Ahmad used symmetric forces and stabilized the system with comparatively low frequency of fast oscillation [14].

\section{Kapitza Method for Arbitrary Periodic Forces}

A classical particle of mass $m$ is moving in time-independent potential field $U$ and a fast oscillating control field. For simplicity, consider one-dimensional motion. Then, the force due to time-independent potential $U(x)$ is

$$
f_{1}(x)=-\frac{d U}{d x}
$$

and a periodic fast oscillating force with zero mean in Fourier series is

$$
f_{2}(x, t)=\sum_{k=1}^{\infty}\left[a_{k}(x) \cos (k \omega t)+b_{k}(x) \sin (k \omega t)\right] .
$$

This fast oscillation has frequency $\omega \equiv 2 \pi / T \gg 2 \pi / T_{U} \equiv \omega_{0}$. Here, $\omega_{0}$ is the frequency of motion due to $f_{1}$. The mean value of a function is denoted by bar and is defined as

$$
\bar{f}=\frac{1}{T} \int_{0}^{T} f(x, t) d t .
$$

Also, the Fourier coefficient $a_{0}$ is

$$
a_{0}(x)=\frac{2}{T} \int_{0}^{T} f_{2}(x, t) d t .
$$

From (3) and (4), it follows that

$$
\bar{f} \cong a_{0}
$$

In (2), $a_{k}$ and $b_{k}$ are the Fourier coefficients given as

$$
\begin{aligned}
& a_{k}(x)=\frac{2}{T} \int_{0}^{T} f_{2}(x, t) \cos k \omega t d t, \\
& b_{k}(x)=\frac{2}{T} \int_{0}^{T} f_{2}(x, t) \sin k \omega t d t .
\end{aligned}
$$


Due to (1) and (2), the equation of motion is

$$
m \ddot{x}=f_{1}(x)+f_{2}(x, t) .
$$

Here at a time two motions are observed: one along a smooth path due to $f_{1}$ and the other small oscillations due to $f_{2}$. So the path can be written as $x(t)=X(t)+\xi(t)$ (see Figure 1). Here, $\xi(t)$ represents small oscillations.

By averaging procedure, the effective potential energy function is [13]

$$
U_{\mathrm{eff}}=U+\frac{1}{4 m \omega^{2}} \sum_{k=1}^{\infty} \frac{\left(a_{k}^{2}+b_{k}^{2}\right)}{k^{2}} .
$$

The pendulum driven by a periodic force is stabilized by minimizing (8). These forces are chosen in the range $[-1,1]$ to establish an exact comparison with harmonic forces. Next, an $\epsilon$-parametric control is developed with one of the driving forces and has better results.

\section{The Pendulum Driven by Harmonic Force}

Consider a pendulum whose point of support oscillates horizontally (see Figure 3), under the influence of harmonic force (Kapitza pendulum). The harmonic force is (see Figure 2)

$$
f(t)=\sin \omega t \quad \text { if } 0 \leq t<T,
$$

and the fast oscillating force is

$$
f_{2}(\phi, t)=m \omega^{2} \cos \phi f(t)
$$

with the meaning $\bar{f}_{2}=0$, which follows from the Fourier coefficient $a_{0}=0$, for (9). The other Fourier coefficients are given by using (6):

$$
\begin{gathered}
a_{k}=0, \\
b_{k}=m \omega^{2} \cos \phi .
\end{gathered}
$$

Then, the forces acting on the particle are

$$
\begin{gathered}
f_{1}=-\frac{d(-m g l \cos \phi)}{d \phi}, \\
f_{2}=m \omega^{2} \cos \phi \sin (\omega t),
\end{gathered}
$$

and the effective potential energy is obtained by using (8):

$$
U_{e f f}=m g l\left(-\cos \phi+\frac{\omega^{2}}{4 g l} \cos ^{2} \phi\right) .
$$

The stable equilibrium is found by minimizing (13). $U_{\text {eff }}$ has the extrema at $\phi=0, \pi, \pm \arccos 2 g l / \omega^{2}$.

(i) The downward point $\phi=0$ is stable if $\omega^{2}<2 g l$.

(ii) Vertically upward point $\phi=\pi$ is not stable.

(iii) The point given by $\cos \phi=2 g l / \omega^{2}$ is stable if $\omega^{2}>2 g l$ [5].

These stable points are illustrated in Figure 4.

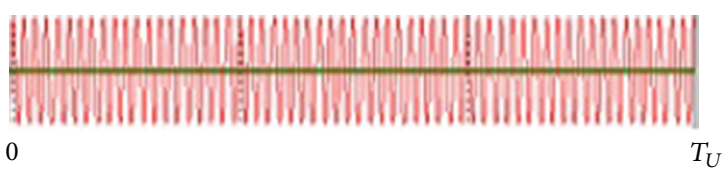

Figure 1: Path of the particle.

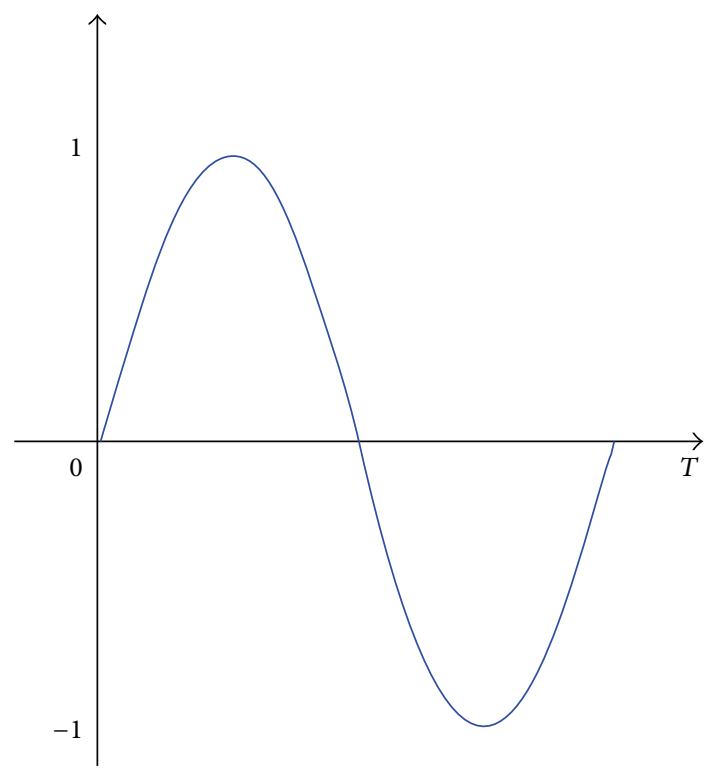

FIGURE 2: Harmonic force.

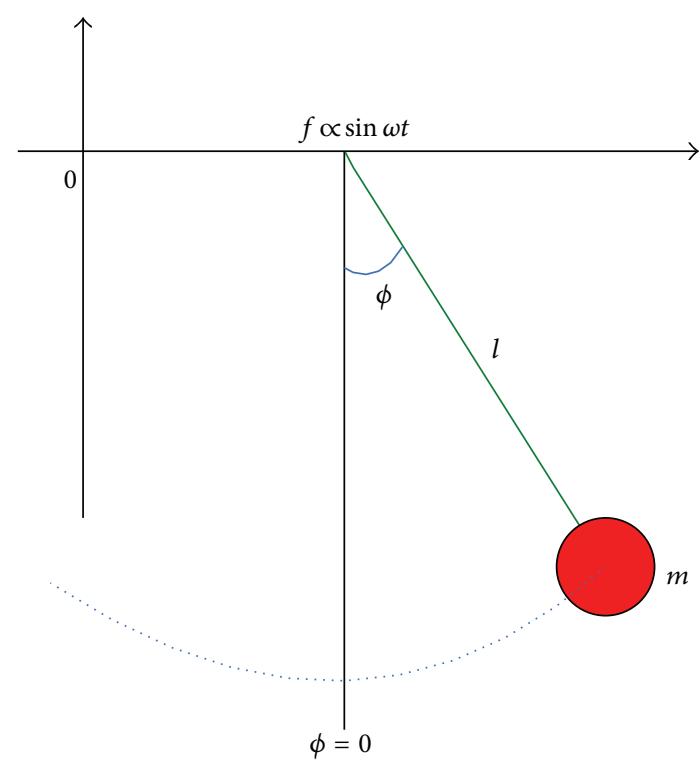

FIGURE 3: Horizontal modulation with harmonic force.

\section{The Pendulum Driven by Periodic Piecewise Linear Forces}

The goal is to stabilize the pendulum with low frequency as compared to harmonic force. Next, this harmonic force is replaced with some periodic piecewise linear forces within 


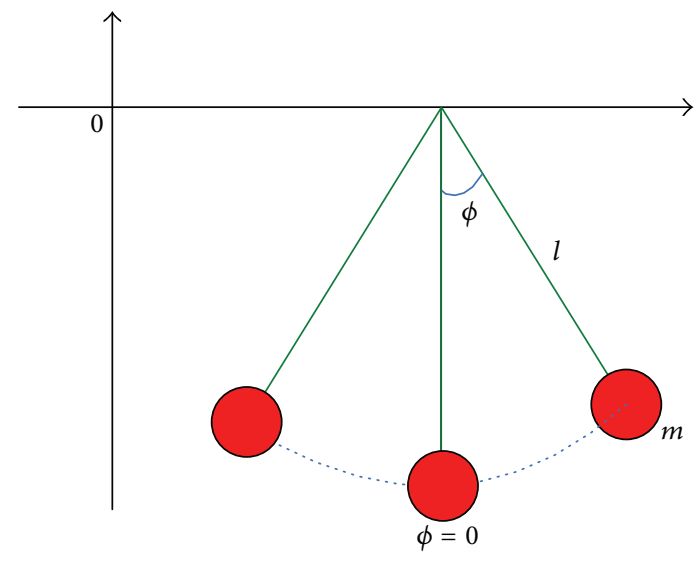

FIgURE 4: Stable points with horizontal oscillations.

the range of harmonic force. These periodic piecewise linear forces are $T$-periodical: $R(t+T, n) \equiv R(t, n)$. For horizontal modulation, the force acting on the particle is

$$
f_{2}(\phi, t)=m \omega^{2} \cos \phi \cdot R(t, n) .
$$

4.1. Triangular Type Force. First of all introduce the triangular type force (see Figure 5) given by

$$
R_{s}(t)= \begin{cases}\frac{4}{T} t & \text { if } 0 \leq t<\frac{T}{4} \\ \frac{4}{T}\left(-t+\frac{T}{2}\right) & \text { if } \frac{T}{4} \leq t<\frac{3 T}{4} \\ \frac{4}{T}(t-T) & \text { if } \frac{3 T}{4} \leq t<T .\end{cases}
$$

For (15), the Fourier coefficient $a_{0}=0$ indicates $\bar{R}_{s}=0$. The Fourier expansion of (14) is

$$
\begin{aligned}
f_{2}(\phi, t)= & m \omega^{2} \cos \phi \frac{8}{\pi^{2}} \\
& \times \sum_{j=0}^{\infty} \frac{(-1)^{j}}{(2 j+1)^{2}} \sin \left(\frac{2 \pi(2 j+1) t}{T}\right) .
\end{aligned}
$$

With

$$
\begin{gathered}
a_{k}=0, \\
b_{k}=m \omega^{2} \cos \phi \frac{8}{\pi^{2}} \frac{(-1)^{j}}{(2 j+1)^{2}},
\end{gathered}
$$

the effective potential energy is

$$
\begin{aligned}
U_{\text {eff }} & =U+m \omega^{2} \cos ^{2} \phi \cdot \frac{1}{4}\left(\frac{8}{\pi^{2}}\right)^{2} \sum_{j=0}^{\infty} \frac{1}{(2 j+1)^{6}} \\
& =U+\frac{\pi^{2}}{60} m \omega^{2} \cos ^{2} \phi
\end{aligned}
$$

which has extrema at $\phi=0, \pi, \pm \arccos 30 \mathrm{gl} / \omega^{2} \pi^{2}$. Minimization of $U_{\text {eff }}$ shows that

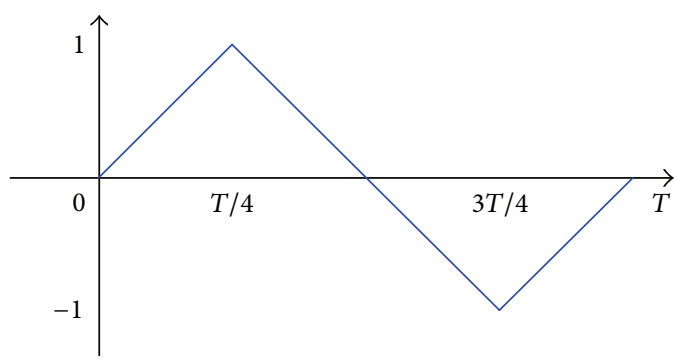

FIGURE 5: Triangular type force.

(i) the downward point $\phi=0$ is stable if $\omega^{2}<3.0396 \mathrm{gl}$,

(ii) vertically upward point $\phi=\pi$ is not stable,

(iii) the point given by $\cos \phi=3.0396 \mathrm{gl} / \omega^{2}$ is stable if $\omega^{2}>3.0396 \mathrm{gl}$.

From (iii), it is observed that, at nontrivial position, the oscillator is stabilized with higher frequency as compared to harmonic force [13].

Hence, this force is less effective than sin- or cos-type force. So, this force is replaced by some other periodic piecewise linear forces.

4.2. Hat Type Force. Now if sine function is traced by linear pulses (see Figure 6), defined by (19)

$$
L_{c}(t)= \begin{cases}\frac{1}{2} & \text { if } 0 \leq t<\frac{1}{6} T, \\ 1 & \text { if } \frac{1}{6} T \leq t<\frac{1}{3} T, \\ \frac{1}{2} & \text { if } \frac{1}{3} T \leq t<\frac{1}{2} T, \\ -\frac{1}{2} & \text { if } \frac{1}{2} T \leq t<\frac{2}{3} T, \\ -1 & \text { if } \frac{2}{3} T \leq t<\frac{5}{6} T, \\ -\frac{1}{2} & \text { if } \frac{5}{6} T \leq t<T,\end{cases}
$$

Next if the sine force is traced by a linear force forming a hat (see Figure 6), defined by (19). For horizontal modulation, the oscillating force is

$$
f_{2}(\phi, t)=m \omega^{2} \cos \phi \cdot L_{c}(t) .
$$

Then, by Fourier expansion in place of (19),

$$
a_{k}=0
$$

$$
b_{k}=m \omega^{2} \cos \phi \frac{1}{k \pi}\left(1-\cos k \pi+2 \cos k \frac{\pi}{3}\right) .
$$

Using the above coefficients, the oscillating force acting on the particle is

$$
\begin{aligned}
f_{2}(t)= & m \omega^{2} \cos \phi \\
& \times \sum_{k=1}^{\infty} \frac{1}{k \pi}\left(1-\cos k \pi+2 \cos k \frac{\pi}{3}\right) \sin k \omega t,
\end{aligned}
$$




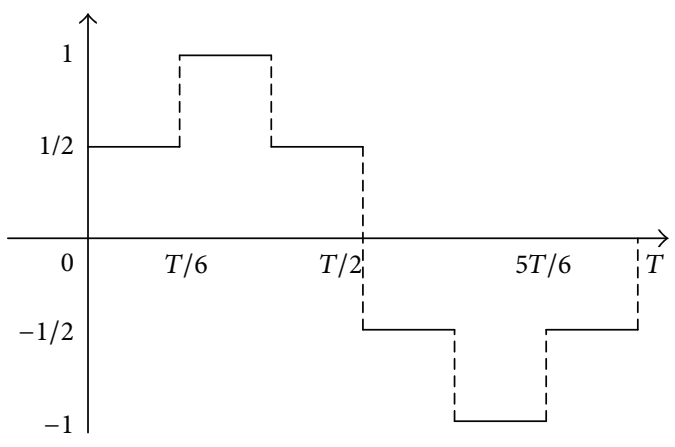

Figure 6: Hat type force.

and the effective potential energy is

$$
\begin{gathered}
U_{\text {eff }}=U+m \omega^{2} \cos ^{2} \phi \\
\cdot \frac{1}{4 \pi^{2}} \sum_{k=1}^{\infty} \frac{1}{k^{2}}\left(1-\cos k \pi+2 \cos k \frac{\pi}{3}\right)^{2} \\
U_{\text {eff }}=U+0.2302 m \omega^{2} \pi^{2} \cos ^{2} \phi
\end{gathered}
$$

which has extrema at $\phi=0, \pi, \pm \arccos 2.172 \mathrm{gl} / \omega^{2}$. Here,

(i) the position $\phi=0$ is stable if $\omega^{2}<2.172 \mathrm{gl}$,

(ii) the inverse position $\phi=\pi$ is not stable,

(iii) the position $\phi=\arccos 2.172 \mathrm{gl} / \omega^{2}$ is stable if $\omega^{2}>$ $2.172 \mathrm{gl}$.

Again a less effective result is obtained. So this periodic piecewise linear force is replaced by another one.

4.3. Trapezium Type Force. If the sine force is traced by a linear shape forming a trapezium (see Figure 7), given by (24)

$$
T_{m}(t)= \begin{cases}\frac{8 t}{T} & \text { if } 0 \leq t<\frac{T}{8} \\ 1 & \text { if } \frac{T}{8} \leq t<\frac{3 T}{8} \\ \frac{8}{T}\left(\frac{T}{2}-t\right) & \text { if } \frac{3 T}{8} \leq t<\frac{5 T}{8} \\ -1 & \text { if } \frac{5 T}{8} \leq t<\frac{7 T}{8} \\ \frac{8(t-T)}{T} & \text { if } \frac{7 T}{8} \leq t<T,\end{cases}
$$

with $\bar{T}_{m}=0$. For horizontal modulation, the force acting on the particle is

$$
f_{2}(\phi, t)=m \omega^{2} \cos \phi \cdot T_{M}(t, n) .
$$

Then, by Fourier expansion in the place of (24),

$$
\begin{gathered}
a_{k}=0, \\
b_{k}=m \omega^{2} \cos \phi \frac{16}{\pi^{2}} \frac{1}{k^{2}} \sin k \frac{\pi}{4} .
\end{gathered}
$$

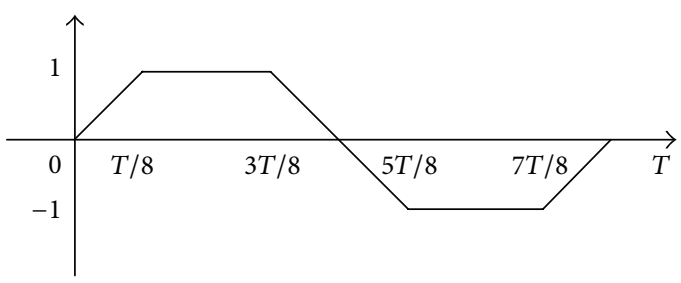

Figure 7: Trapezium type force.

Using the above coefficients, the oscillating force is

$$
\begin{aligned}
f_{2}(\phi, t)= & m \omega^{2} \cos \phi \frac{16}{\pi^{2}} \\
& \times \sum_{k=0}^{\infty} \frac{1}{k^{2}} \sin k \frac{\pi}{4} \sin k \omega t
\end{aligned}
$$

and the effective potential energy is

$$
\begin{aligned}
U_{\text {eff }}= & U+m \omega^{2} \cos ^{2} \phi \cdot \frac{1}{4}\left(\frac{16}{\pi^{2}}\right)^{2} \\
& \times \sum_{k=0}^{\infty} \frac{1}{k^{6}} \sin ^{2} k \frac{\pi}{4} \\
= & U+0.3393 m \omega^{2} \cos ^{2} \phi
\end{aligned}
$$

which has extrema at $\phi=0, \pi, \pm \arccos 1.4736 \mathrm{gl} / \omega^{2}$. Here,

(i) the point $\phi=0$ is stable if $\omega^{2}<1.4736 \mathrm{gl}$,

(ii) the point $\phi=\pi$ is not stable,

(iii) the point given by $\cos \phi=1.4736 \mathrm{gl} / \omega^{2}$ is stable if $\omega^{2}>1.4736 \mathrm{gl}$.

From (iii), it is observed that, at nontrivial position, the oscillator is stabilized with lower frequency as compared to harmonic force. So this type of force is much effective than sin- or cos-type force. Next, modify this trapezium shape force to have a better result.

4.4. Quadratic Type Force. If slopes are removed in the beginning and at the end from it and define a quadratic type force: $Q_{c}(t)=Q_{c}(t+T)$ (see Figure 8), given by (29)

$$
Q_{c}(t)= \begin{cases}1 & \text { if } 0 \leq t<\frac{3 T}{8} \\ \frac{8}{T}\left(\frac{T}{2}-t\right) & \text { if } \frac{3 T}{8} \leq t<\frac{5 T}{8} \\ -1 & \text { if } \frac{5 T}{8} \leq t<T,\end{cases}
$$




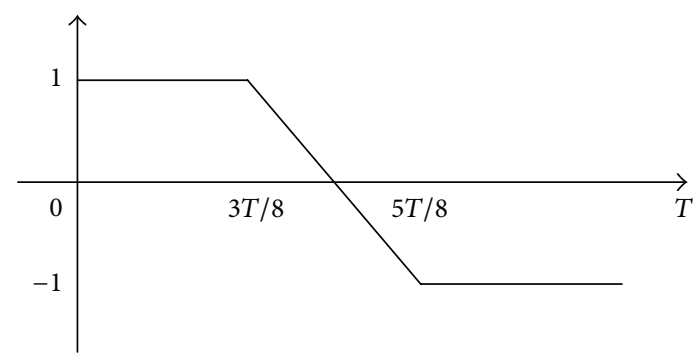

FIGURE 8: Quadratic type force.

with the same property $\bar{Q}_{c}=0$. For horizontal modulation, the oscillating force is

$$
f_{2}(\phi, t)=m \omega^{2} \cos \phi \cdot Q_{c}(t, n) .
$$

The Fourier expansion of (30) is

$$
\begin{aligned}
Q_{c}(t)= & m \omega^{2} \cos \phi \\
& \times \sum_{k=0}^{\infty}\left(\frac{2}{k \pi}+\frac{8}{\pi^{2} k^{2}} \sin k \frac{\pi}{4}\right) \sin k \omega t,
\end{aligned}
$$

and the effective potential energy is

$$
\begin{aligned}
U_{\text {eff }}= & U+m \omega^{2} \cos ^{2} \phi \\
& \cdot \frac{1}{4} \sum_{k=0}^{\infty} \frac{1}{k^{2}}\left(\frac{2}{k \pi}+\frac{8}{\pi^{2} k^{2}} \sin k \frac{\pi}{4}\right)^{2} \\
= & U+0.3856 m \omega^{2} \cos ^{2} \phi
\end{aligned}
$$

which has extrema at $\phi=0, \pi, \pm \arccos 1.2967 \mathrm{gl} / \omega^{2}$. Here,

(i) the position $\phi=0$ is stable if $\omega^{2}<1.2967 \mathrm{gl}$,

(ii) the position $\phi=\pi$ is not stable,

(iii) the position $\phi=\arccos 1.2967 \mathrm{gl} / \omega^{2}$ is stable if $\omega^{2}>$ $1.2967 \mathrm{gl}$.

Again the frequency of oscillation is lower at nontrivial position.

4.5. Rectangular Type Force. Now if we introduce rectangular type force: $R_{l}(t)=R_{l}(t+T)$ (see Figure 9 ), given by (33)

$$
R_{l}(t)= \begin{cases}1 & 0 \leq t \leq \frac{T}{2} \\ -1 & \frac{T}{2} \leq t \leq T\end{cases}
$$

with the same property $\bar{R}_{l}=0$. For horizontal modulation, the force acting on the particle is

$$
f(t)=m \omega^{2} \cos \phi \cdot R_{l}(t, n),
$$

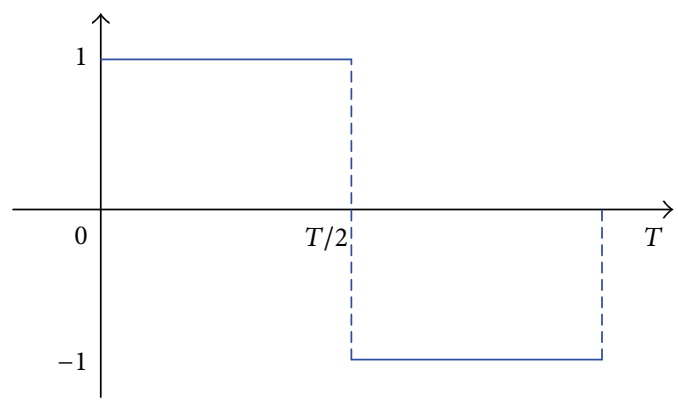

FIGURE 9: Rectangular type force.

and its Fourier expansion is

$$
\begin{aligned}
R_{l}(t)= & m \omega^{2} \cos \phi \frac{4}{\pi} \\
& \times \sum_{k=0}^{\infty} \frac{1}{(2 k-1)} \sin (2 k-1) \omega t ;
\end{aligned}
$$

the effective potential energy is

$$
\begin{aligned}
U_{\text {eff }}= & U+m \omega^{2} \cos ^{2} \phi \\
& \cdot \frac{1}{4}\left(\frac{16}{\pi^{2}}\right)^{2} \sum_{k=0}^{\infty} \frac{1}{(2 k-1)^{4}} \\
= & U+0.4112 m \omega^{2} \cos ^{2} \phi
\end{aligned}
$$

which has extrema at $\phi=0, \pi, \pm \arccos 1.2159 \mathrm{gl} / \omega^{2}$. Here,

(i) the point $\phi=0$ is stable if $\omega^{2}<1.2159 \mathrm{gl}$,

(ii) the point $\phi=\pi$ is not stable,

(iii) the point $\phi=\arccos 1.2159 \mathrm{gl} / \omega^{2}$ is stable if $\omega^{2}>$ $1.2159 \mathrm{gl}[13,14]$.

From (iii), it is observed that, at nontrivial position with the help of this type of external force, the frequency of oscillation has become much lower. At nontrivial position, the above results are summarized in Table 1. From these results, it is also observed that, as the area under the curve increases, the frequency of oscillation decreases, at nontrivial position. The triangular type force has minimum area and so has maximum frequency, while rectangular type force has maximum area and has minimum frequency.

\section{Vertical Modulation}

For vertical modulation with harmonic force (see Figure 10), the fast oscillating force is

$$
f_{2}=m \omega^{2} \sin \phi \cdot \sin \omega t .
$$

Here, the position $\phi=0$ is always stable, and the inverse point $\phi=\pi$ is stable if $\omega^{2}>2 g l$ (see Figure 11) [5]. 
TABLE 1: Stability comparison of periodic piecewise linear forces with harmonic force under horizontal fast oscillation.

\begin{tabular}{lcccc}
\hline Force type & Trivial position & Stability condition & Nontrivial position & Stability condition \\
\hline Sin & 0 & $\omega^{2}<2 g l$ & $\pm \arccos 2 g l / \omega^{2}$ & $\omega^{2}>2 g l$ \\
Triangular & 0 & $\omega^{2}<3.0396 g l$ & $\pm \arccos 3.0396 g l / \omega^{2}$ & $\omega^{2}>3.0396 g l$ \\
Linear (sine) & 0 & $\omega^{2}<2.172 g l$ & $\pm \arccos 2.172 g l / \omega^{2}$ & $\omega^{2}>2.172 g l$ \\
Trapezium & 0 & $\omega^{2}<1.4736 g l$ & $\pm \arccos 1.4736 g l / \omega^{2}$ & $\omega^{2}>1.4736 g l$ \\
Quadratic & 0 & $\omega^{2}<1.2967 g l$ & $\pm \arccos 1.2967 g l / \omega^{2}$ & $\omega^{2}>1.2967 g l$ \\
Rectangular & 0 & $\omega^{2}<1.2159 g l$ & $\pm \arccos 1.2159 g l / \omega^{2}$ & $\omega^{2}>1.2159 g l$ \\
\hline
\end{tabular}

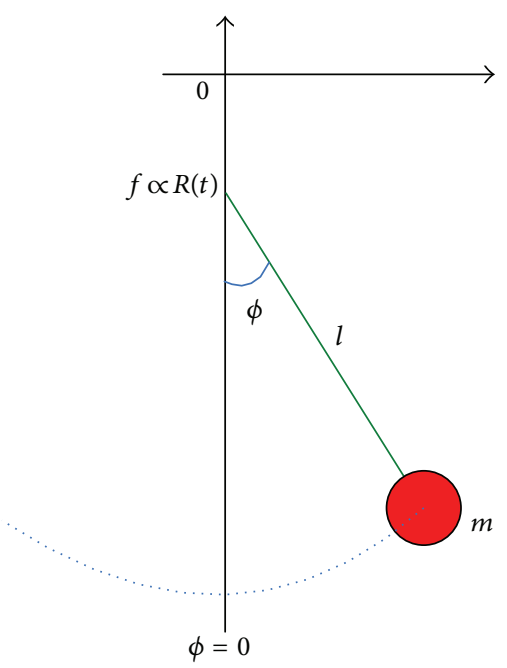

Figure 10: Kapitza pendulum with vertical oscillation.

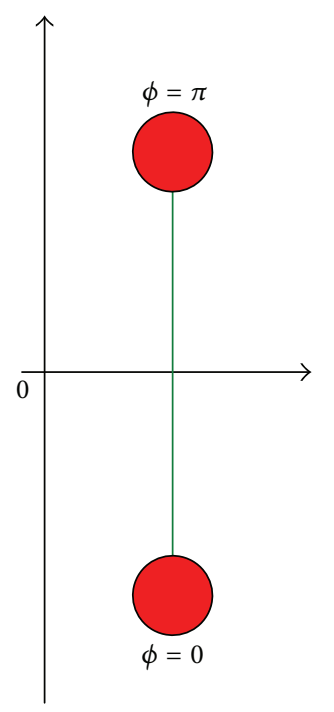

FIGURE 11: Stable points for vertical oscillation.

Using external periodic piecewise linear forces, (37) takes the form

$$
f=m \omega^{2} \sin \phi \cdot R(t, n),
$$

where $R(t, n)$ is the external periodic piecewise linear forces. The stability results at $\phi=\pi$ are summarized in Table 2 .
TABLE 2: Stability comparison of periodic piecewise linear forces with harmonic force under vertical fast oscillation.

\begin{tabular}{lcccc}
\hline Force type & Position & $\begin{array}{c}\text { Stability } \\
\text { condition }\end{array}$ & Position & Stability condition \\
\hline Sin & 0 & Always & $\pi$ & $\omega^{2}>2 g l$ \\
Triangular & 0 & Always & $\pi$ & $\omega^{2}>3.0396 g l$ \\
Linear (sine) & 0 & Always & $\pi$ & $\omega^{2}>2.172 g l$ \\
Trapezium & 0 & Always & $\pi$ & $\omega^{2}>1.4736 g l$ \\
Quadratic & 0 & Always & $\pi$ & $\omega^{2}>1.2967 g l$ \\
Rectangular & 0 & Always & $\pi$ & $\omega^{2}>1.2159 g l$ \\
\hline
\end{tabular}

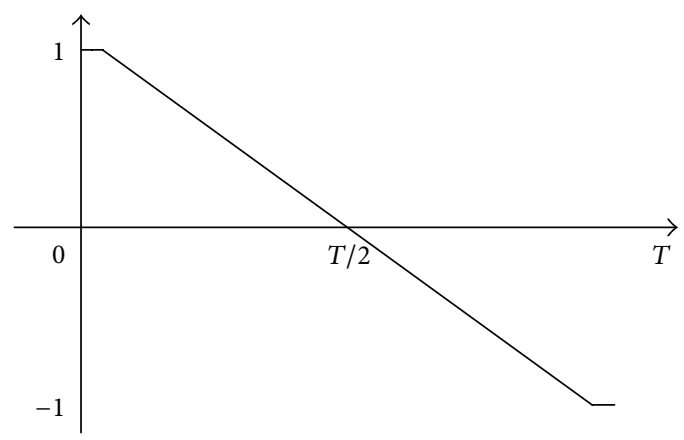

FIgURe 12: $\epsilon$-Parametric quadratic type force.

\section{Parametric Control}

All the above results can be considered as nonparametric control. Next, an $\epsilon$-parametric control is defined for one of the periodic piecewise linear forces. At nontrivial position, the frequency of oscillation is calculated. This $\epsilon$-parametric force with $0<\epsilon<1$ is given by (similar to external force (29))

$$
R_{\epsilon}(t)= \begin{cases}1 & \text { if } 0 \leq t<\frac{1-\epsilon}{2} T, \\ \frac{1}{\epsilon}\left(-\frac{2}{T} t+1\right) & \text { if } \frac{1-\epsilon}{2} T \leq t<\frac{1+\epsilon}{2} T \\ -1 & \text { if } \frac{1+\epsilon}{2} T \leq t<T\end{cases}
$$

and illustrated in Figure 12. 
The Fourier coefficient $a_{0}=0$ indicates $\bar{R}_{\epsilon}=0$. For horizontal modulation, the oscillating force acting on the particle is

$$
f_{2}(\phi, t)=m \omega^{2} \cos \phi \cdot R_{\epsilon}(t, n) .
$$

With (39), the Fourier coefficients are

$$
\begin{gathered}
a_{k}=0 \\
b_{k}=m \omega^{2} \cos \phi\left(\frac{2}{(k \pi)}+\frac{2}{\epsilon k^{2} \pi^{2}} \sin \epsilon k \pi\right),
\end{gathered}
$$

and the oscillating force in fourier expansion is

$$
\begin{aligned}
f_{2}(t)= & m \omega^{2} \cos \phi \\
& \times \sum_{k=1}^{\infty}\left(\frac{2}{(k \pi)}+\frac{2}{\epsilon k^{2} \pi^{2}} \sin \epsilon k \pi\right) \sin k \omega t .
\end{aligned}
$$

The effective potential energy is

$$
\begin{aligned}
U_{\text {eff }}= & U+m \omega^{2} \cos ^{2} \phi \\
& \cdot \frac{1}{4 \pi^{2}} \sum_{k=1}^{\infty} \frac{4}{k^{4}}\left(1+\frac{1}{\epsilon k \pi} \sin \epsilon k \pi\right)^{2} \\
= & -m g l \cos \phi+m \omega^{2} \cos ^{2} \phi \cdot b,
\end{aligned}
$$

where

$$
b=\frac{1}{\pi^{2}} \sum_{k=1}^{\infty} \frac{1}{k^{4}}\left(1+\frac{1}{\epsilon k \pi} \sin \epsilon k \pi\right)^{2}
$$

which has extremum at $\phi=0, \pi, \pm \arccos 0.5 \mathrm{gl} / \omega^{2} b$.

The stability of the system is discussed under the force with different values of $\epsilon$. See Figure 13. First of all consider $\epsilon=0.9$; the infinite sum is

$$
b=0.1320,
$$

and the effective potential energy is

$$
U_{\text {eff }}=-m g l \cos \phi+0.132 m \omega^{2} \cos ^{2} \phi .
$$

The nontrivial position $\pm \arccos \left(3.7879 \mathrm{gl} / \omega^{2}\right)$ is stable under the condition $\omega^{2}>3.7879 \mathrm{gl}$. This value is larger than the above considered examples, such a poor result. Next for $\epsilon=0.8$, the infinite sum is 0.1607 , and the nontrivial position $\pm \arccos \left(3.1114 \mathrm{gl} / \omega^{2}\right)$ is stable if $\omega^{2}>3.1114 \mathrm{gl}$, such a better result. Also, it is found that, as $\epsilon$ decreases, the infinite sum $b$ increases and the system is stabilized with a relatively low frequency. For different values of $\epsilon$, the results of infinite sum $b$ and the nontrivial position $\pm \arccos 0.5 \mathrm{gl} / \omega^{2} b$ with stable condition are given in Table 3 .

Also as $\epsilon \rightarrow 0$, the term $b \cong 0.4386$, and the position $\pm \arccos \left(1.14 \mathrm{gl} / \omega^{2}\right)$ is stable under the condition $\omega^{2}>1.14 \mathrm{gl}$ which is lower than with rectangular type force. Hence, with parametric control, the rectangular type force is approached, and the system is stabilized with a relatively low frequency.

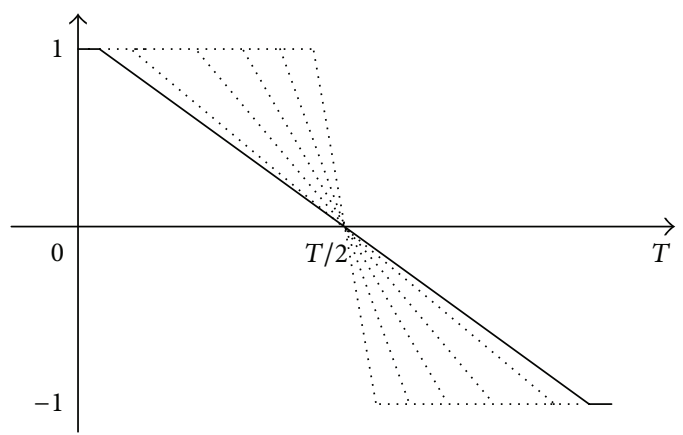

FIgURE 13: Quadratic type force with different $\epsilon(0.9-0.1)$.

TABLE 3: Stability conditions with $\epsilon$-parametric force.

\begin{tabular}{lccc}
\hline$\epsilon$ & Sum & Nontrivial position & Stability condition \\
$0<\epsilon<1$ & $b$ & & \\
\hline 0.9 & 0.1320 & $\pm \arccos \left(3.7879 \mathrm{gl} / \omega^{2}\right)$ & $\omega^{2}>3.7879 \mathrm{gl}$ \\
0.8 & 0.1607 & $\pm \arccos \left(3.1114 \mathrm{gl} / \omega^{2}\right)$ & $\omega^{2}>3.1114 \mathrm{gl}$ \\
0.75 & 0.1775 & $\pm \arccos \left(2.8169 \mathrm{gl} / \omega^{2}\right)$ & $\omega^{2}>2.8169 \mathrm{gl}$ \\
0.7 & 0.1956 & $\pm \arccos \left(2.5562 \mathrm{gl} / \omega^{2}\right)$ & $\omega^{2}>2.5562 \mathrm{gl}$ \\
0.6 & 0.2357 & $\pm \arccos \left(2.1213 \mathrm{gl} / \omega^{2}\right)$ & $\omega^{2}>2.1213 \mathrm{gl}$ \\
0.5 & 0.2793 & $\pm \arccos \left(1.7902 \mathrm{gl} / \omega^{2}\right)$ & $\omega^{2}>1.7902 g l$ \\
0.4 & 0.3239 & $\pm \arccos \left(1.5437 \mathrm{gl} / \omega^{2}\right)$ & $\omega^{2}>1.5437 g l$ \\
0.3 & 0.3664 & $\pm \arccos \left(1.3647 \mathrm{gl} / \omega^{2}\right)$ & $\omega^{2}>1.3647 \mathrm{gl}$ \\
0.25 & 0.3856 & $\pm \arccos \left(1.2967 \mathrm{gl} / \omega^{2}\right)$ & $\omega^{2}>1.2967 g l$ \\
0.2 & 0.4029 & $\pm \arccos \left(1.241 \mathrm{gl} / \omega^{2}\right)$ & $\omega^{2}>1.241 \mathrm{gl}$ \\
0.1 & 0.4287 & $\pm \arccos \left(1.1663 \mathrm{gl} / \omega^{2}\right)$ & $\omega^{2}>1.1663 g l$ \\
\hline
\end{tabular}

The minimization of dimensionless effective potential energy function with horizontal modulation is shown in Figure 14 and with vertical modulation is shown in Figure 15.

Here, the same effect is also observed; as $\epsilon$ decreases, the area under the curve increases, and the value of $b$ increases; consequently, the frequency of oscillation becomes low at nontrivial position. In this connection, an interesting result is obtained; when $\epsilon \rightarrow 0$, the quadratic type force approaches the rectangular type force, so at nontrivial position the frequency of oscillation should be almost the same, but, with parametric force, the frequency of oscillation is low. Observe from Table 3, the rectangular force fall between $\epsilon=0.2$ and $\epsilon=0.1$, more clearly, the parametric force with $\epsilon=$ $0.17 \ldots$, gives the frequency of oscillation almost equals with rectangular type force, hence comparatively less area shows low frequency at non-trivial position.

\section{Conclusions}

Using Kapitza method of averaging for an arbitrary periodic force, the modulated pendulum with periodic piecewise linear force is stabilized with frequency $\omega$ that is sufficiently lesser than that in the case of harmonic modulation. In this contest, rectangular force was found to be the best. But this force is more effective when it has a time-dependent 


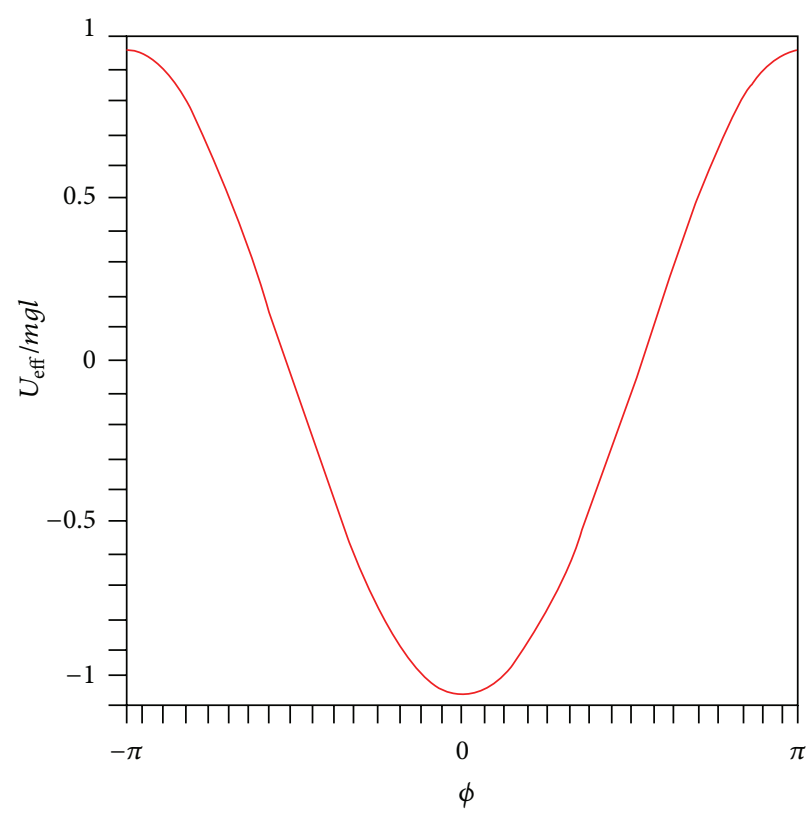

(a) $U_{\text {eff }}$ is minimum at $\phi=0$ if $\omega^{2}<1.14 \mathrm{gl}$

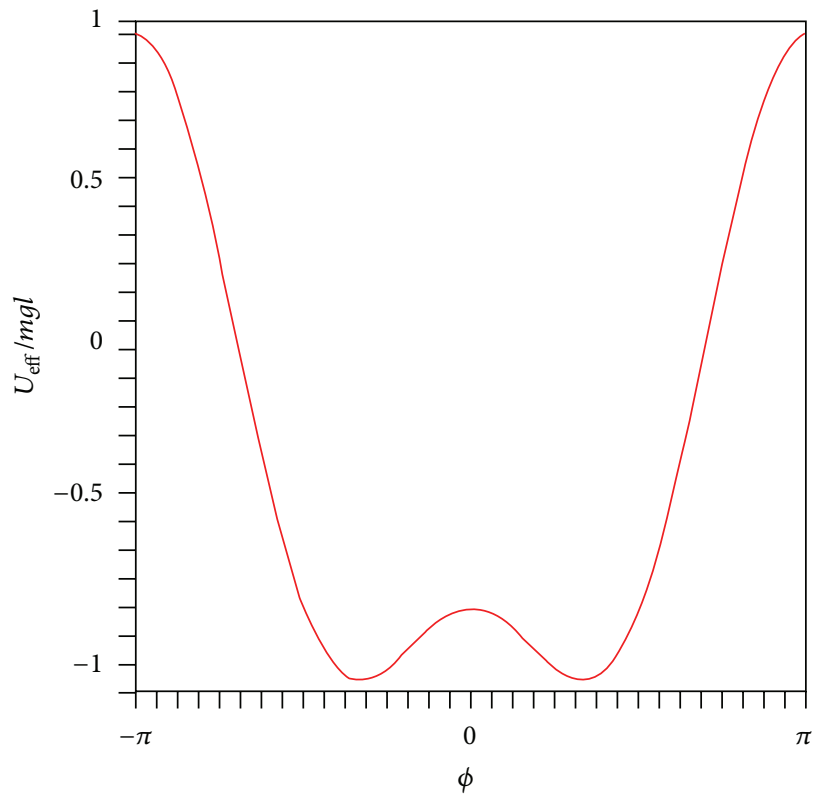

(b) $U_{\text {eff }}$ is minimum at $\cos \phi=1.2159 \mathrm{gl} / \omega^{2}$ if $\omega^{2}>1.14 \mathrm{gl}$

FIGURE 14: Horizontal oscillation.

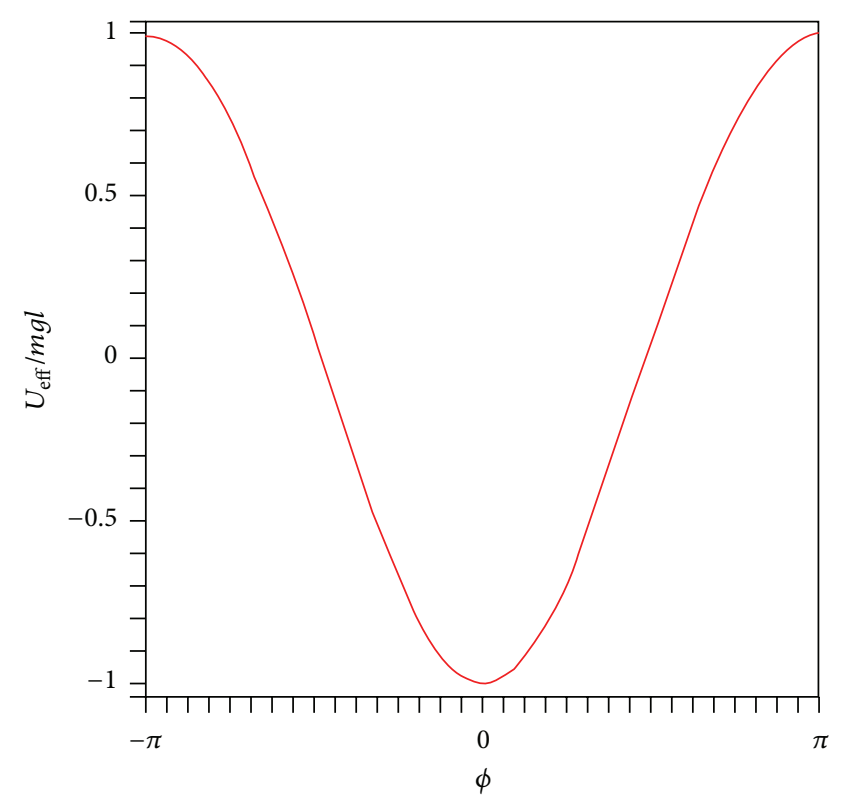

(a) $U_{\text {eff }}$ is always minimum at $\phi=0$

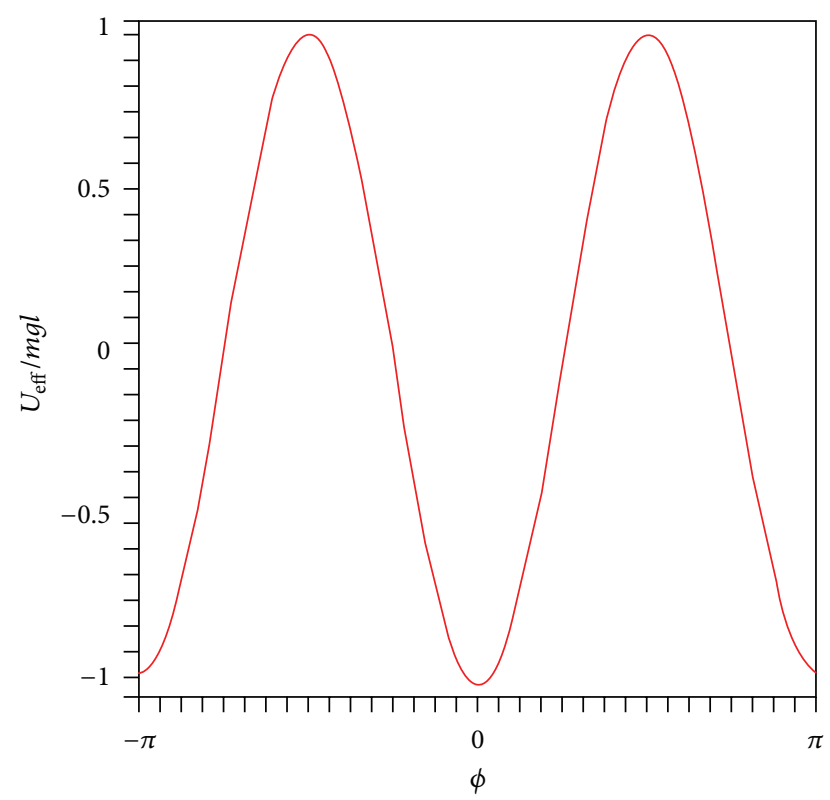

(b) $U_{\text {eff }}$ is minimum at $\phi=\pi$ if $\omega^{2}>1.14 \mathrm{gl}$

Figure 15: Vertical oscillation.

structure. This time-dependent structure is found by defining a parametric control on some other periodic piecewise linear forces. Hence, a more suitable form of rectangular force is found.

The parametric control can be applied to control the nontrivial stable position, for horizontally or vertically modulated pendulum.

\section{References}

[1] A. Stephenson, "On a new type of dynamic stability," Memories and Proceeding of the Manchester Literary and Philosophical Society, vol. 52, pp. 1-10, 1908.

[2] A. Stephenson, "On induced stability," Philosophical Magazine, vol. 15, pp. 233-236, 1908. 
[3] A. Stephenson, "On induced stability," Philosophical Magazine, vol. 17, pp. 765-766, 1909.

[4] P. L. Kapitza, "Dynamic stability of a pendulum with an oscillating point of suspension," Journal of Experimental and Theoretical Physics, vol. 21, pp. 588-597, 1951.

[5] L. D. Landau, E. M. Lifshitz, and Mecanics, vol. 15, Pergamon Press: butterworth, Oxford, UK, 3rd edition, 2005.

[6] L. S. Brown, "Quantum motion in a Paul trap," Physical Review Letters, vol. 66, no. 5, pp. 527-529, 1991.

[7] W. Paul, "Electromagnetic traps for charged and neutral particles," Reviews of Modern Physics, vol. 62, no. 3, pp. 531-540, 1990.

[8] I. Gilary, N. Moiseyev, S. Rahav, and S. Fishman, "Trapping of particles by lasers: the quantum Kapitza pendulum," Journal of Physics A, vol. 36, no. 25, pp. L409-L415, 2003.

[9] F. Bullo, "Averaging and vibrational control of mechanical systems," SIAM Journal on Control and Optimization, vol. 41, no. 2, pp. 542-562, 2003.

[10] Y. Nakamura, T. Suzuki, and M. Koinuma, "Nonlinear behavior and control of a nonholonomic free-joint manipulator," IEEE Transactions on Robotics and Automation, vol. 13, no. 6, pp. 853862, 1997.

[11] J. A. Hołyst and W. Wojciechowski, "The effect of Kapitza pendulum and price equilibrium," Physica A, vol. 324, no. 1-2, pp. 388-395, 2003.

[12] A. L. Fradkov, "Application of cybernetic methods in physics," Physics-Uspekhi, vol. 48, no. 2, pp. 103-127, 2005.

[13] B. Ahmad and S. Borisenok, "Control of effective potential minima for Kapitza oscillator by periodical kicking pulses," Physics Letters A, vol. 373, no. 7, pp. 701-707, 2009.

[14] B. Ahmad, "Stabilization of Kapitza oscillator by symmetric periodical forces," Nonlinear Dynamics, vol. 62, no. 3, pp. 499506, 2010. 

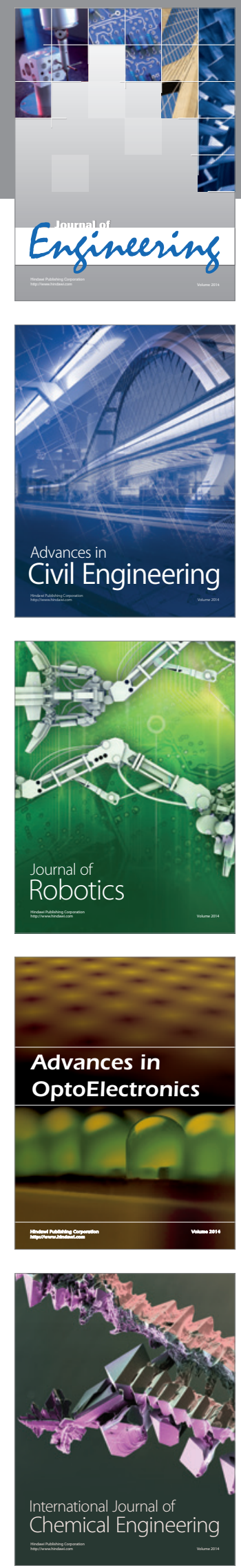

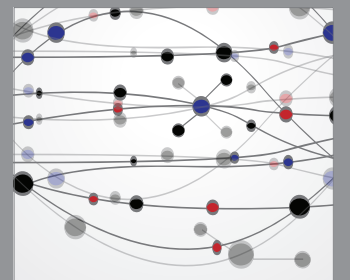

The Scientific World Journal
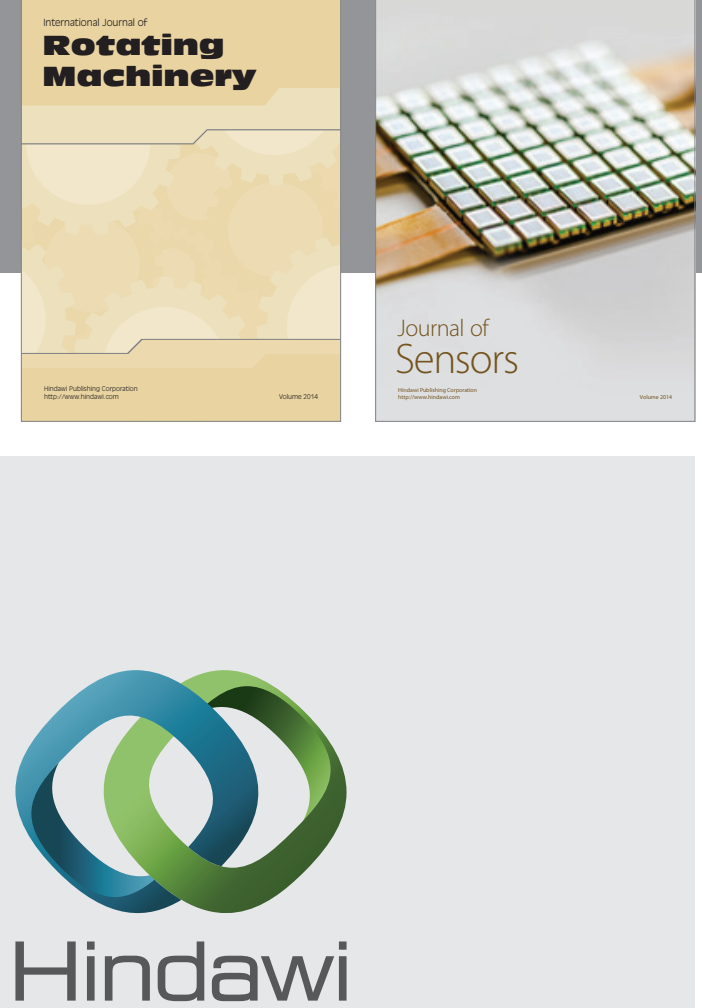

Submit your manuscripts at http://www.hindawi.com
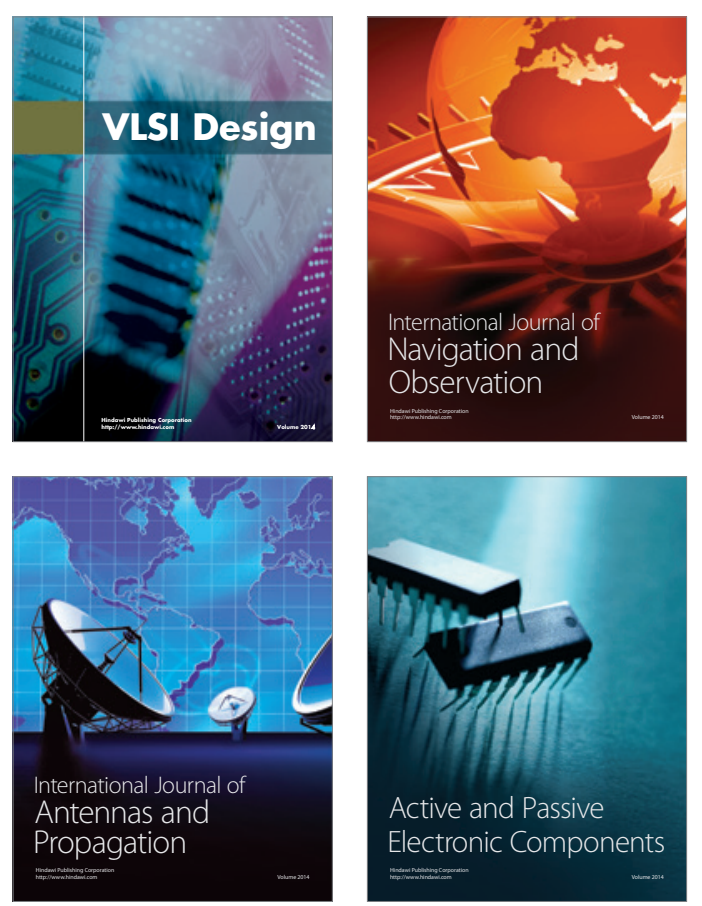
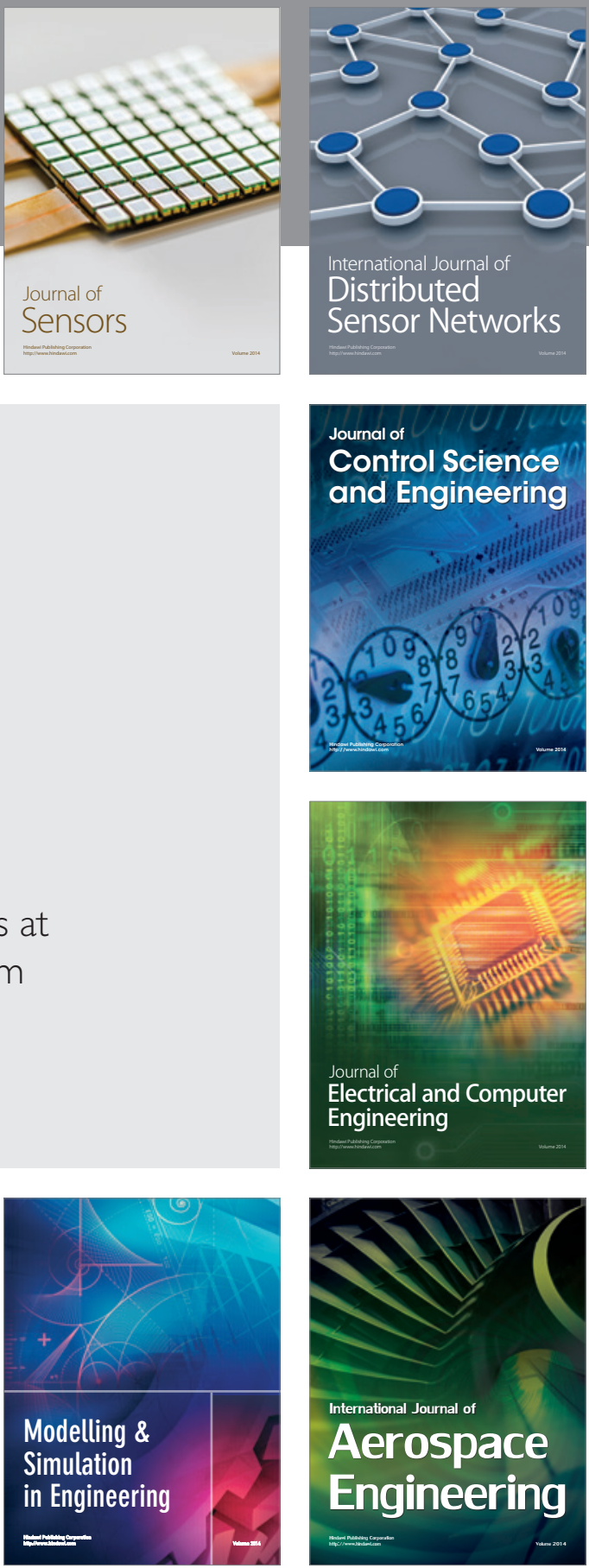

Journal of

Control Science

and Engineering
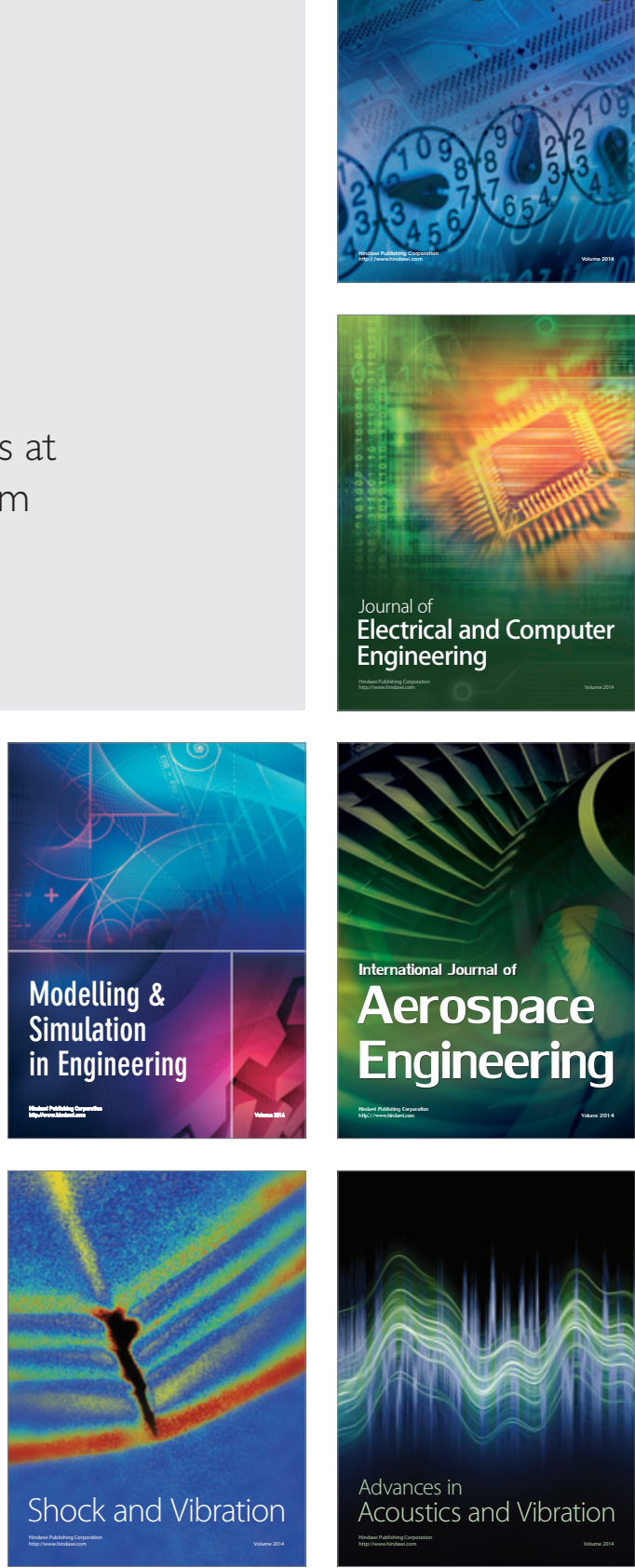